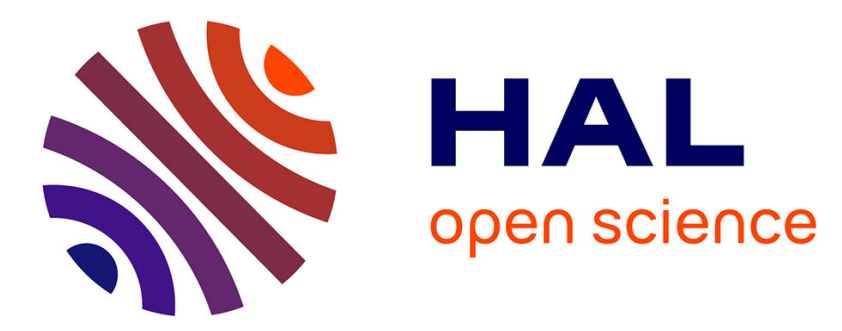

\title{
A classifying registration technique for the estimation of enhancement curves of DCE-CT scan sequences
}

Mohamed Hachama, Agnes Desolneux, Charles A Cuenod, Frédéric Richard

\section{To cite this version:}

Mohamed Hachama, Agnes Desolneux, Charles A Cuenod, Frédéric Richard. A classifying registration technique for the estimation of enhancement curves of DCE-CT scan sequences. Medical Image Analysis, 2010, 14 (2), pp.185-194. 10.1016/j.media.2009.12.002 . hal-00308358

\section{HAL Id: hal-00308358 \\ https://hal-univ-paris.archives-ouvertes.fr/hal-00308358}

Submitted on 30 Jul 2008

HAL is a multi-disciplinary open access archive for the deposit and dissemination of scientific research documents, whether they are published or not. The documents may come from teaching and research institutions in France or abroad, or from public or private research centers.
L'archive ouverte pluridisciplinaire HAL, est destinée au dépôt et à la diffusion de documents scientifiques de niveau recherche, publiés ou non, émanant des établissements d'enseignement et de recherche français ou étrangers, des laboratoires publics ou privés. 


\section{A CLASSIFYING REGISTRATION TECHNIQUE FOR THE ESTIMATION OF ENHANCEMENT CURVES OF DCE-CT SCAN SEQUENCES}

MOHAMED HACHAMA, FRÉDÉRIC J.P. RICHARD, DANIEL BALVAY, ROKHAYA THIAM, AGNÈS DESOLNEUX, AND CHARLES A. CUENOD

CORRESPONDING AUTHOR: Frédéric RICHARD.

\section{AUTHOR INFORMATION.}

Mohamed HACHAMA, Frédéric RICHARD and Agnès DESOLNEUX

University Paris Descartes,

MAP5, CNRS UMR 8145,

Department of Mathematics,

Tel. : +331-44-553541,

Fax. : +331-42-864144,

(hachama,richard,desolneux)@math-info.univ-paris5.fr

and,

Daniel BALVAY, Rokhaya THIAM and Charles CUÉNOD

University Paris Descartes,

LRI-EA4062

Hospital Georges Pompidou,

Service of Radiology.

Tel.: +331-56-093841,

Fax.: +331-56-093850

ca@cuenod.net

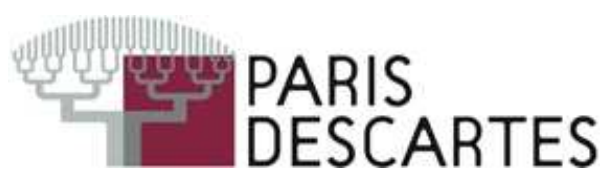

Date: July 30, 2008. 


\begin{abstract}
In this paper, we propose a new technique for the estimation of contrast enhancement curves of Dynamic Contrast Enhanced sequences, which takes the most from the interdependence between this estimation problem and the registration problem raised by possible movements occuring in sequences. The technique solves the estimation and registration problems simultaneously in an iterative way. However, unlike previous techniques, a pixel classification scheme is included within the estimation so as to compute enhancement curves on pixel classes instead of single pixels. The classification scheme is designed using a descendant hierarchical approach. Due to this tree approach, the number of classes is set automatically and the whole technique is entirely unsupervised. Moreover, some specific prior information about the form of enhancement curves are included in the splitting and pruning steps of the classification scheme. Such an information ensures that created classes include pixels having homogeneous and relevant enhancement properties. The technique is applied to DET-CT scan sequences and evaluated using ground truth data. Results show that classifications are anatomically sound and that constrast enhancements are accurately estimated from sequences. Moreover, due to the classification scheme, the curve estimation is more robust to the sequence noise than pixel-based estimations and the computation time is significantly lower.
\end{abstract}

Keywords: Image registration ; Dynamic Contrast-Enhanced images ; Enhancement curves estimation.

\title{
1. INTRODUCTION
}

Last years have witnessed an increase in the use of dynamic imaging modalities such as Dynamic Contrast-Enhanced Computed Tomography (DCE-CT) [1] and Magnetic Resonance Imaging (DCE-MRI) [2]. Such modalities generate time series of $2 \mathrm{D}$ or $3 \mathrm{D}$ images of human organs, which are relevant for the diagnosis and the management of cancers $[3,4,5,6]$, ischemic diseases such as stroke [7], myocardial ischemia [8], and inflammation. In such sequences, a contrast agent is used to distinguish and enhance different organ tissues which are characterized by their absorption of contrast agent through time $[9,10]$.

However, the clinical analysis of these images usually requires computer processing tools facilitating the image interpretation. As related to contrast agent concentration in tissue, a temporal intensity evolution of a single or a group of pixels, called enhancement curve, is usually used for the automation of lesion detection and diagnosis $[11,12]$. Such curves are also used to define parametric maps of some extracted parameters to get a functional segmentation of images. However, the analysis of enhancement curves faces two main problems: the presence of noises and movements of patients.

Sequences of DCE CT-scans have a poor signal-to-noise ratio due to the limited irradiation dose used for sequential acquisition and to tomographic reconstruction error. Hence, there is a need to denoise enhancement curves obtained directly from images. For that, some authors proposed to estimate the contrast enhancement curves using some mathematical prior models which define possible forms of curves 
$[13,11,14,15,16]$. There are two main classes of prior models. The first class contains models derived from a pharmacokinetic modeling of the contrast agent concentration and circulation in two interacting compartments: namely, blood plasma and extra-vascular-extracellular space EES $[13,11,14]$. Obtained models depend on some parameters which are assumed to be linked to the physiological process and can be estimated using statistical techniques $[17,11,14]$. The second class of models is based on the use of heuristic parametric curves which reproduce main features of an expected curve such as the base-line signal, the enhancement rate, the time to peak, the peak of enhancement, or the final slope [15, 16, 12]. This second class is more generic and does not require any assumption or inference about the underlying physiology.

Since the acquisition of a DCE sequence takes some minutes, patient movements and physiologic motion (caused, for instance, by breathing, heartbeat, peristalsis) cannot be avoided and generate normal differences between images which must be corrected before any curve analysis. Image registration, which maps images in a common space, is usually applied to correct geometric differences between images. But, in most classical registration techniques, a same relation between pixel intensities is assumed for the whole image [18]. Such an assumption implies that two pixels with similar intensities in an image have similar intensities in another image. This hypothesis is not valid in many cases. In DCE images, normal tissues and lesions have different enhancements after injection even though their intensities are similar before injection. One can also observe dynamic enhancement differences between benign and malignant tumors. In other words, the contrast enhancement varies locally depending on the tissue type and not exclusively on initial intensities.

In [19], Rougon et al. adapted the Mutual Information criterion for the registration of a first pre-contrast injection image to a second post-contrast image. Assuming that the first image can be segmented into some regions having homogeneous enhancement properties, they defined a regionalized criterion based on mutual information on each region. Such an approach requires a preliminary segmentation of images. But, in our DET-CT problem, it is difficult to obtain such a segmentation before image registration. The sequence segmentation is to be done according to dynamic enhancement properties of pixels, which are better estimated using the registered images. In other words, not only the sequence registration requires taking into account a local information about pixel enhancements but also the estimation of enhancements needs the sequence to be registered. Therefore, the enhancement estimation and the registration are two interdependent problems.

For that reason, some authors proposed to solve the two problems simultaneously. In [11], Hayton et al. used a pharmacokinetic model to describe enhancement for each pixel $x$ by a temporal curve $C_{x}(t)$. They used an optical flow-based technique for the registration of each image of an original sequence observed at time $t$ to an ideal image $I^{t}$ where $I^{t}(x)=C_{x}(t)$ for all pixels $x$. In their technique, registration 
and estimation are iterated successively: registered images are used for the enhancement curve estimation and the estimated curves are used to generate ideal images $I^{t}(x)$ to which original images are registered. This technique was applied for the improvement of lesion detection in mammography. A similar idea was used in [14] for the registration of axial volumes within the abdomen to synthetic volumes built upon estimated enhancement curves. As in [11], enhancement curves are estimated locally on each pixel. Such an estimation is both time-consuming and noise sensitive. In [20], Xiaohua et al. proposed a Bayesian framework which is based on a Maximum A Posteriori estimation and performs simultaneously registration of breast MRI images and segmentation of non-fat tissues into three regions: normal tissue, benign lesions and malignant lesions. In this work, authors estimate dynamic enhancement curves and classify pixels depending on some features computed using enhancement curves (early-phase enhancement rate, and intermediate and final slope). Since curve estimation is done for each pixel, their technique presents the same drawbacks as those in $[14,11]$. Moreover, the number of classes has to be set manually.

In this paper, we propose a new technique for the estimation of enhancement curves of DCE-CT scan sequences. This technique takes the most from the interdependence between this estimation problem and the registration problem posed by possible movements occuring in sequences. Similarly to $[14,11,20]$, it solves the estimation and registration problems simultaneously in an iterative way. However, the main departure of our techniques from previous ones is the use of a pixel classification scheme within the estimation procedure. Due to the introduction of this scheme, enhancement curves can be estimated on pixel classes instead of single pixels. This new feature makes the enhancement estimation more robust to noise than in pixel-based techniques and reduces the computation time.

The classification scheme is designed using a descendant hierarchical approach. Due to this tree approach, the number of classes is set automatically and the whole technique can be entirely unsupervised. Moreover, the classification scheme includes some specific knowledges about the form of enhancement curves. Enhancement curves estimated on classes are described using heuristic parametric curves similar to those used in $[15,16,12]$. This description of class curves is used in the splitting and pruning steps of the classification scheme. It enforces classes to correspond to parts of the sequence having homogeneous and realistic enhancement properties.

\section{Method}

Let $\Omega$ be a continuous domain, $\Omega_{d}$ an associated discrete grid on which images are observed $(\operatorname{Card}(\Omega)=N)$, and $J^{t}$ the image of the original sequence observed at time $t$. Our technique aims to estimate from the original sequence $\left(J^{t}\right)_{t \in T}$, an ideal image sequence $I$ where the noise is attenuated and patient movements are compensated. The ideal sequence, which will be called the "temporal template", is defined as a real-value function on $\Omega \times T$. 
2.1. Template definition. We assume that pixels of the domain can be partitionned into $K$ homogeneous classes having specific dynamic enhancements: $\Omega_{d}=$ $\cup_{k=1}^{K} \Omega^{k}$. Such an hypothesis is true in our medical application where pixels belonging to the same tissue type have similar enhancement properties. To introduce some priors on dynamic enhancement curves, we further assume that these curves belong to a class of heuristic models [15], which have the following form:

$$
C(t)=\frac{p_{2}+p_{5} t}{1+e^{\left(-p_{4}\left(s-p_{3}\right)\right)}}+p_{1} .
$$

In such models, the parameters $\left(p_{i}\right)_{1 \leq i \leq 5}$ charaterize the curve form. On Figure 1, we show an example of the fitting of the heuristic model (1) to an empirical curve computed from images.

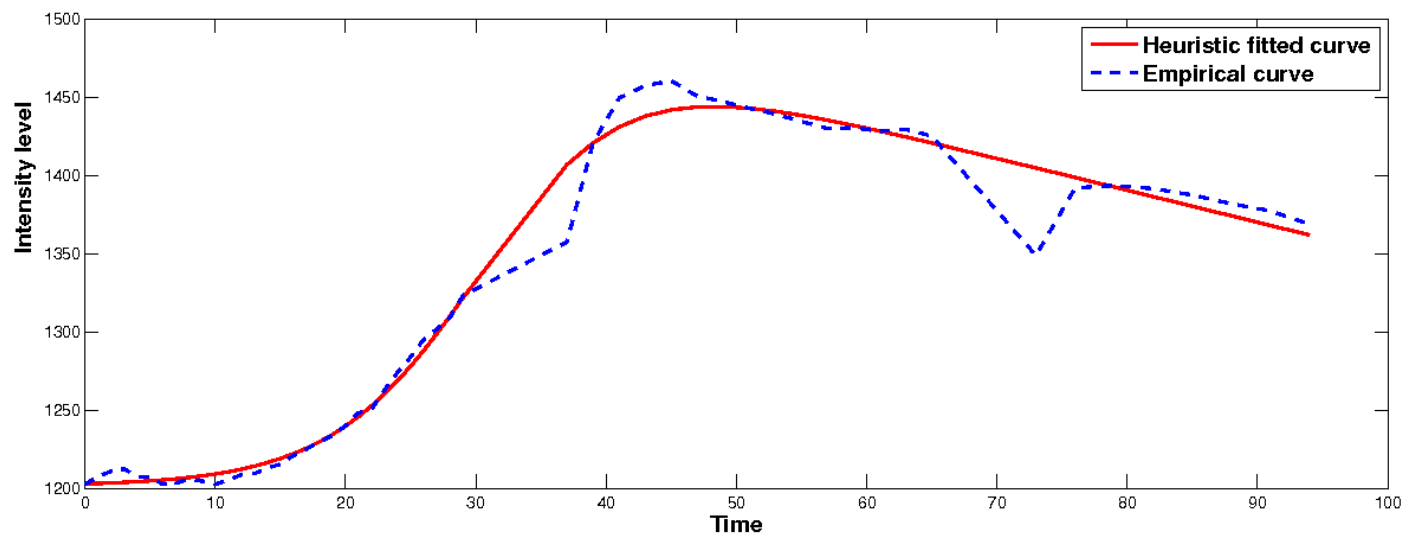

FiguRE 1. Fitting the heuristic model (1) to an empirical curve.

Taking into account both pixel classes and their associated enhancement curves, denoted by $\left(C^{k}\right)_{k}$, the temporal template can be defined for all $t \in T, k \in\{1, \ldots, K\}$ and $x \in \Omega^{k}$ as

$$
I(x, t)=C^{k}(t)=\frac{p_{2}^{k}+p_{5}^{k} t}{1+\exp \left(-p_{4}^{k}\left(t-p_{3}^{k}\right)\right)}+p_{1}^{k} .
$$

We will denote by $I^{t}=I(., t)$ the template frame at time $t$, which will be registered to the observed image $J^{t}$. The deformation making the two images similar will be denoted by $\phi^{t}\left(I^{t} \circ \phi^{t} \sim J^{t}\right)$.

2.2. Technique description. On the one hand, the estimation of the temporal template requires a movement correction by image registration. On the other hand, the image registration uses the temporal template so as to take into account contrast changes. As described on Figure 2, our technique enables to perform the two tasks (estimation and registration) simultaneously by iterating two interacting blocks, called blocks (R) and (T). 


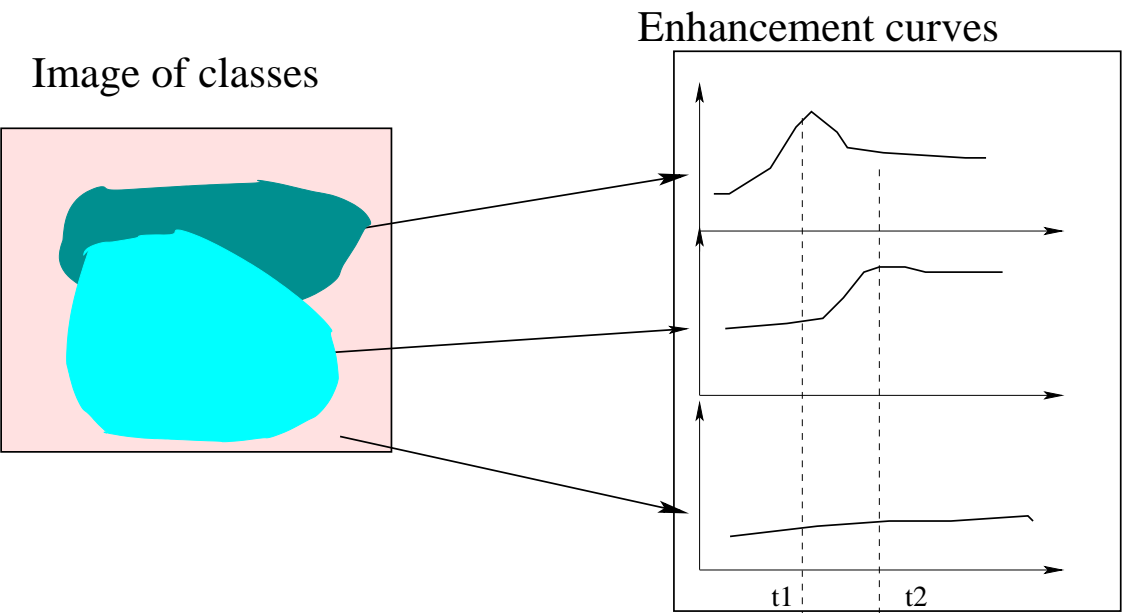

Template at time $\mathrm{t} 1$

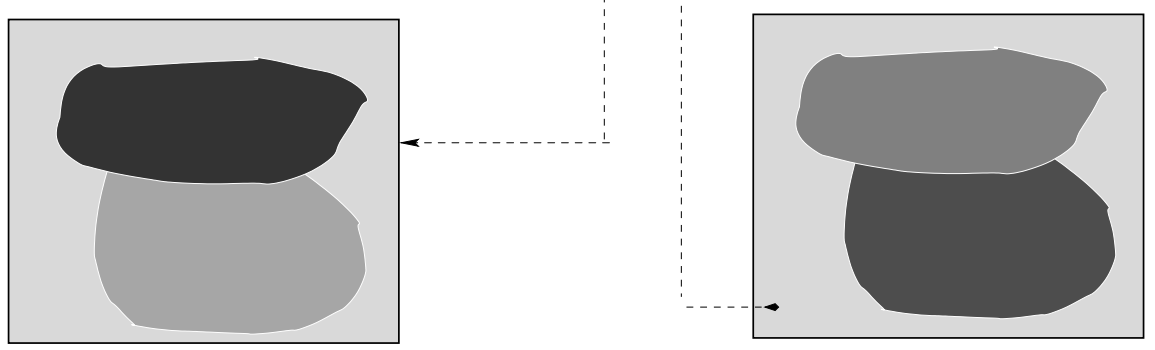

Figure 2. The temporal template construction based on pixel classes and associated enhancement curves.

The block $(R)$ concerns the image registration. The temporal template $I$ estimated from the block $(T)$ is registered to observed images $\left(J^{t}\right)_{t}$, frame by frame (i.e. $I^{t}$ is registered to $J^{t}$ for all $t$ ). The output of the block $(R)$ is a set of deformations $\left(\phi^{t}\right)_{t \in T}$ obtained from registrations and defined later. These deformations are the input of the block $(T)$, which is devoted to the temporal template estimation. The block $(T)$ combines a pixel classification and an estimation of enhancement curve of each class. The pixel features used in the classification are the pixel enhancement curves $\left(C_{x}\right)_{x \in \Omega_{d}}$ defined for all $t$ in $T$ as

$$
C_{x}(t)=J^{t}\left(\left(\phi^{t}\right)^{-1}(x)\right),
$$

where $\left(\phi^{t}\right)^{-1}$ is the inverse map of $\phi$. These local curves estimated at each pixel position are different from the class curves $\left(C^{k}\right)_{k=1}^{K}$ (also called class centers), which are of the parametric form (1). The two blocks are further presented in next paragraphs.

2.3. Block (T). In this section, we describe the combined classification and estimation technique used for the estimation of the temporal template. Assuming that some deformations $\phi^{t}$ are given for all $t$, pixels $x \in \Omega_{d}$ are classified according to 


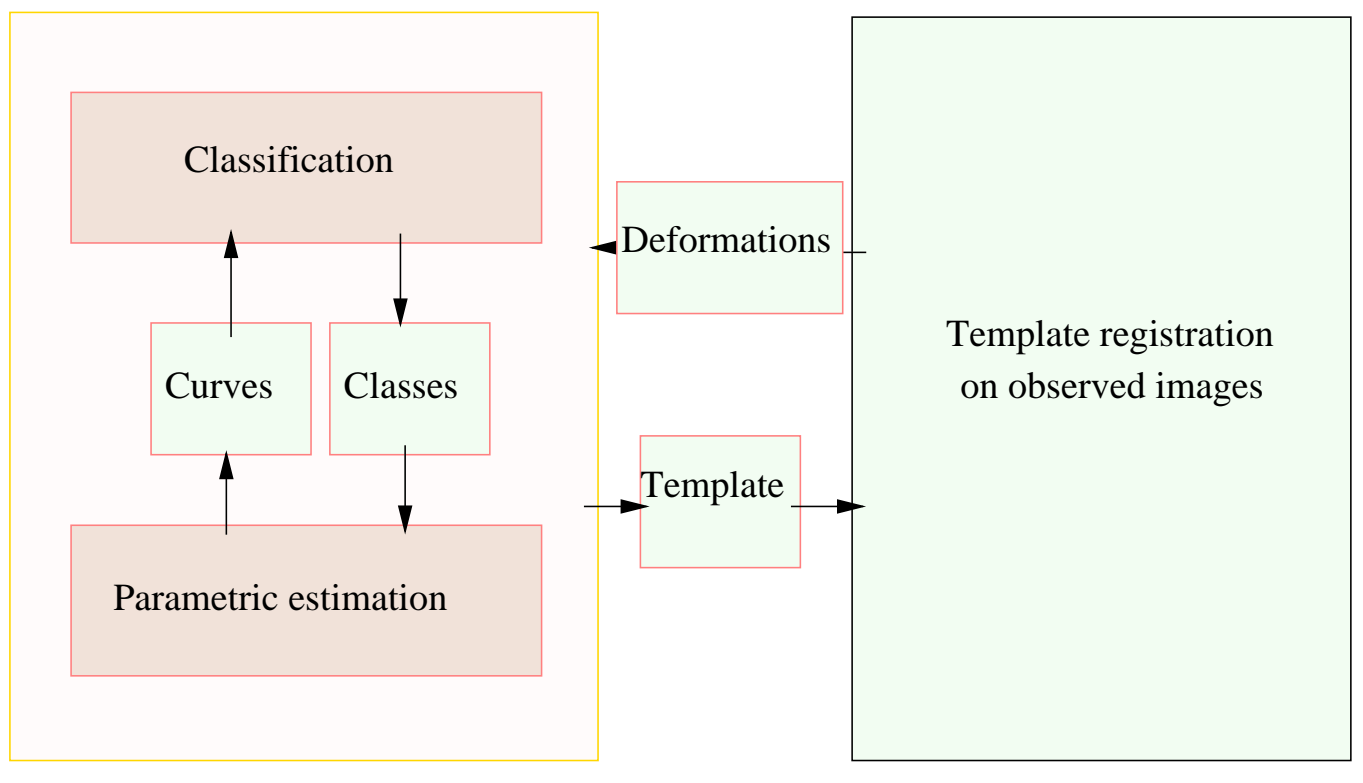

Bloc T

Bloc R

Figure 3. The two inteacting blocks of the technique: the image registration block $(\mathrm{R})$ and the temporal template estimation $(\mathrm{T})$. The technique interleaves a pixel classification and an estimation of the enhancement curve for each class.

their associated pixel enhancement curves $\left(C_{x}\right)_{x \in \Omega_{d}}$ (Equation 3). The classification technique is composed of three successive steps (an initialization step, a tree classification and a pruning step) described next.

2.3.1. Initialization step. First, we have to initialize the technique by setting some initial classes. For that, one could use a manual classification established by an expert, or an ad hoc classification containing one single class if no prior information is available. In our application (axial abdominal and thoracic CT scans), we however apply a simple thresholding procedure to the first image of the sequence in order to fix three initial classes ('air','compact bone', and 'others'). In the first image, compact bones regions have the highest intensities whereas air regions have the lowest. Hence, both classes can be easily detected using both low and high thresholds. Threshold values are fixed for all CT-scan experiments according to the Hounsfield scale ( -1000 for the air, and 4000 for the compact bones). In addition, since these classes do not absorb contrast agent, their intensities tend to remain constant with time. Hence, centers of these classes are set as constants.

2.3.2. Tree classification. In this step, we use a top-down hierarchical classification. As illustrated on Figure 4, this technique generates a tree structure of embedded pixel classes by splitting classes successively at different levels of the tree. The initial classification obtained in the first step is associated to the superior level (level 1). 


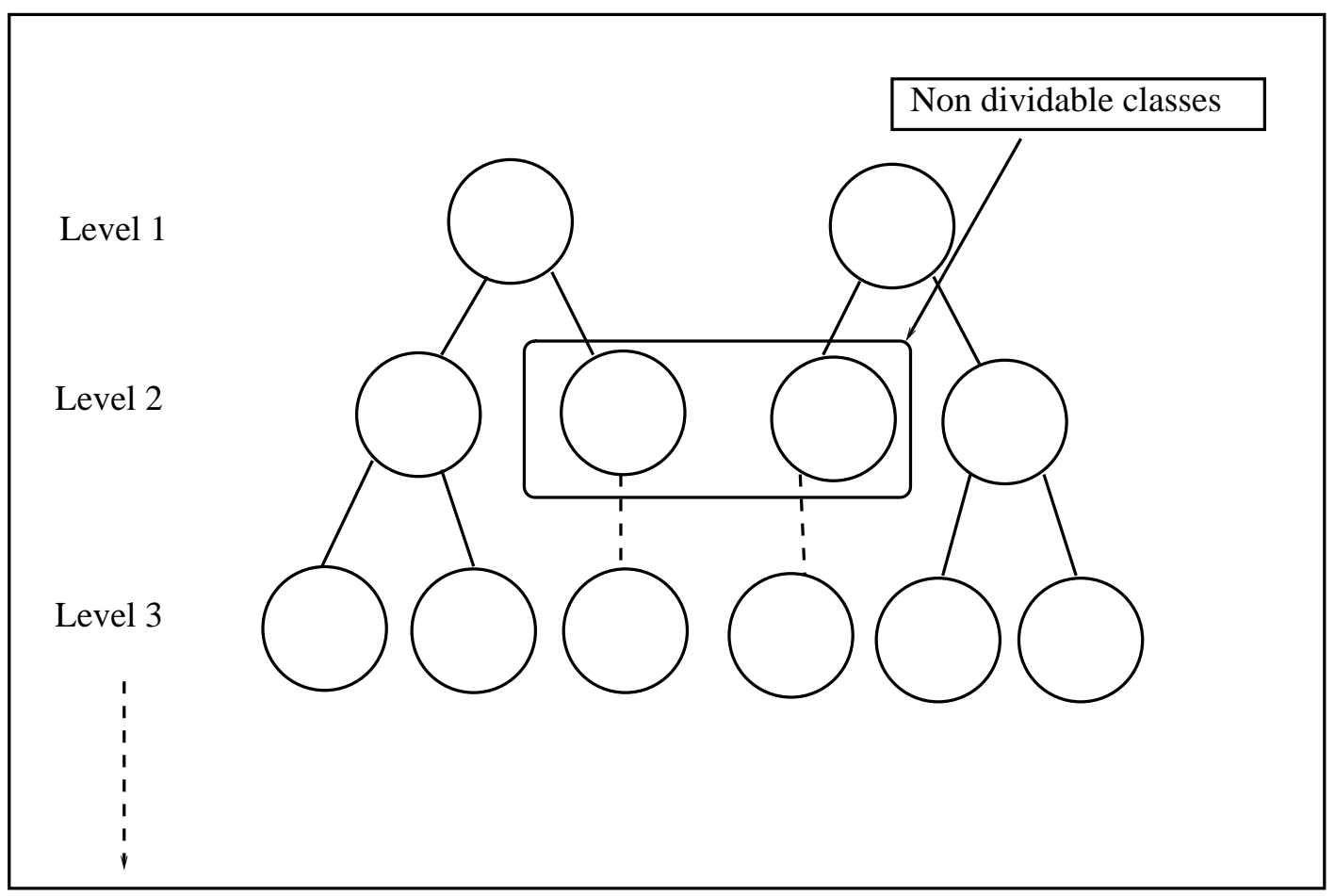

FiguRE 4. Descendant hierarchical classification

Assuming that the technique has built the $h$ highest levels, the level $h+1$ of the tree is then constructed as follows. Each class of the level $h$ is first divided into two candidate clusters using a parametric K-means, which will be described next. Then, if a candidate class is well separated from all other classes, it is accepted as a new class and the class of level $h$ which it originates from is removed. The criterion used for defining a distance between class is the minimum between the class centers reached among all times. A class is considered well-separated from another class if the distance is below a threshold, which is fixed once for all according to the noise variance and the number of images in the sequence.

For building the classification tree, we modified the usual K-means technique in order to take into account some prior knowledges about the class centers. The modified technique iterates two successive steps until convergence.

(1) Compute each class center by fitting a parametric curve of the form (1) to all enhancement curves of pixels of the class (or, equivalently, to their arithmetic average) according to a least square criterion.

(2) Classify each pixel into the nearest class according to distances between class centers and to the enhancement curve associated to the pixel.

2.3.3. Pruning step. At the end of the construction of the tree, we have a pruning step to remove possible remaining inconsistent classes. A pixel class with an enhancement curve $C$ is considered as inconsistent when its mean contrast change is 
negative:

$$
\sum_{t}(C(t)-C(\min (T)))<0 .
$$

Here we used a simple criterion which can be completed with other medically-based criteria. When a class is removed, its pixels are affected to the remaining class for which the distance between the center and the pixel enhancement curve is the lowest.

2.4. Image registration. So as to register a template frame $I^{t}$ to a corresponding observed image $J^{t}$, we compute a deformation $\phi^{t}$, defined as a mapping of $\Omega$ onto $\Omega$ minimizing an energy of the form

$$
E\left(\phi^{t}\right)=R\left(\phi^{t}\right)+S\left(J^{t}, I\right)=R\left(\phi^{t}\right)+\sum_{i=1, \cdots, N}\left[J^{t}\left(x_{i}\right)-I\left(\phi^{t}\left(x_{i}\right), t\right)\right]^{2},
$$

where $R$ is an elastic potential

$$
R\left(\phi^{t}\right):=\frac{1}{2} \int_{\Omega}\left(\sum_{i=1}^{2} \sum_{j=1}^{2} \frac{\lambda}{2}\left(\frac{\partial u_{i}^{t}(x)}{\partial x_{i}}\right)\left(\frac{\partial u_{j}^{t}(x)}{\partial x_{j}}\right)+\frac{\mu}{4}\left(\frac{\partial u_{i}^{t}(x)}{\partial x_{j}}+\frac{\partial u_{j}^{t}(x)}{\partial x_{i}}\right)^{2}\right) d x .
$$

Constants $\lambda$ and $\mu$, which are called the Lame coefficients in Elasticity, are set once for all in all experiments $\left(\lambda=10^{-15}\right.$ and $\left.\mu=20\right)$ and $u^{t}=\phi^{t}-I d$ are displacements associated to deformations. The second term $S$ of the energy expresses some similarity constraints between observed images and template frames, which implies that images from observations and template have same intensities at each time $t$. Such an assumption is garanteed if the template includes a good estimation of contrast changes observed in images. The registration of all images is obtained by minimizing the following energy

$$
E\left(\phi^{1}, \ldots, \phi^{T}\right)=\operatorname{sum}_{t=1}^{T}\left(R\left(\phi^{t}\right)+\sum_{i=1}^{N}\left[J^{t}\left(x_{i}\right)-I\left(\phi^{t}\left(x_{i}\right), t\right)\right]^{2}\right) .
$$

For the discretization of this optimisation problem, we use the finite element technique (Polynomial Lagrange elements $P 1$ ) to approximate the continuous deformations by a finite combination of local functions [21]. We then apply a gradient descent technique on the set of coefficients defining this combination. So as to speed up the technique convergence, we have a multigrid approach, which consists of first initializing deformations with coarse approximation using a small number of local functions and then increasing the number of local functions whenever necessary. In practice, we used from 150 to 4300 local functions. At each resolution, we apply the classification/estimation once and iterate the gradient descent of the image registration until convergence.

The implementation of the classification/estimation block is done using Matlab while the one of the registration block is done using FREEFEM++, which permits to easily handle resolution changes. 


\section{Results}

We applied our technique to several sequences of DCE-CT scans. Here, we present some typical results obtained on sequences of two different sections, namely the upper abdomen and the thorax. Some images of these sequences are presented on first columns of Figures 5 and 6.

Images were acquired at the same axial level with an in-plane resolution of $512 \times 512$ pixels. For the abdominal sequence (53 images), images were acquired at three uniformly sampled intervals of time, corresponding to periods of apnea. For the thoracic sequence, images were acquired every seconds after the contrast agent injection. We only used the first 30 images, in which the main intensity variations are observed. In addition, an iodinated contrast media was injected to enhance the vascular properties of tissues. The anatomy underlying the sequences is briefly described on Figure 7.

The contrast agent injection enables to enhance and observe some classes which are not visible before injection, e.g. veins and tumors. However, images suffer from a poor signal-to-noise ratio, due to the limited irradiation dose used for the sequential acquisition (acquisition parameters were fixed at $80 \mathrm{Kv}$ and $50 \mathrm{mAs}$ ). Besides, observed tissues were deformed during the sequence, due to the patient breathing, heart pulsations, gastric contractions and some possible voluntary movements.

On Figures 8 and 9, we show classifications and associated enhancement curves obtained before and after the application of our technique. Enhancement curves result from averaging gray-level values of pixels of each class at each time. They are shown in Hounsfield Units (UH).

In the first sequence, enhancement curves drawn before registration have peaks at 35 and 75 seconds, corresponding to the patient respiration after periods of apnea. These peaks are well attenuated by the registration. In the second sequence, we notice that enhancement curves which corresponds to different tissue classes are well separated after registration. For instance, after registration, we can distinguish four classes of pixels which contrast curves are among the highest. Those classes correspond to the arch of aorta, the superior vena cava and compact bones. This anatomical segmentation is obtained due to the creation of interface classes whose initial intensities vary between -400 and 0 , notably, associated to lungs borders. As shown on the first sequence, the technique obtained several classes of pixels on the tumor and on the fail (see Figure 8). Such a result is anatomically sound because (1) the tumor is composed of vascular and cell parts having different dynamic behaviors and (2) the fail is a tissular component composed of red and white pulps with different vascularisations. The algorithm also obtained two pixel classes for the vena cava (see Figure 9). This can be explained by the fact that, when they arrive in the vena cava, the contrast agent and the blood are not mixed yet, and have different dynamic behaviors. Those classification results suggest that pixel classifications are consistent with the anatomy and that the technique is accurate enough to distinguish 

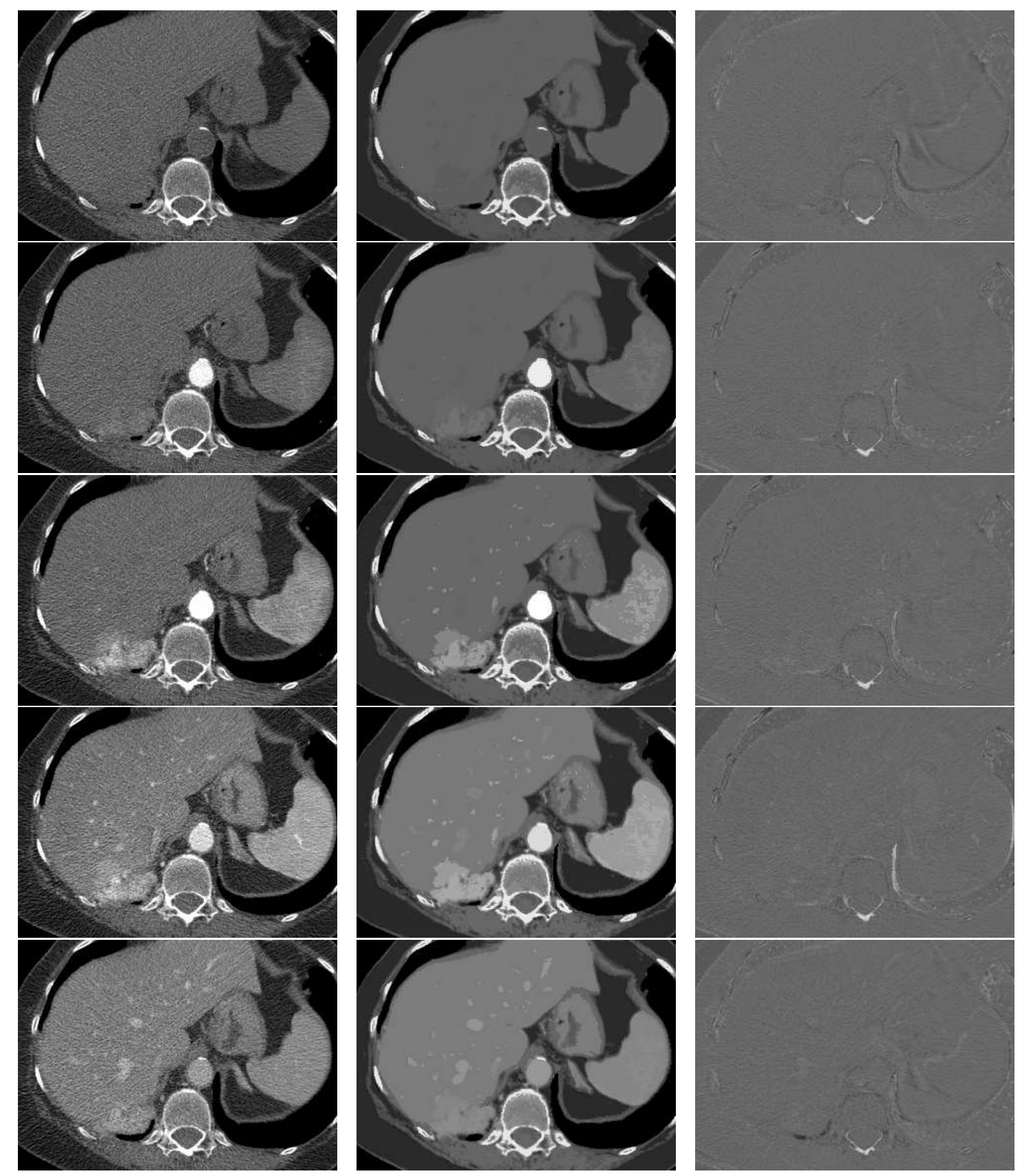

Figure 5. An abdominal DET-CT scan sequence. Some images of the original sequence (1st column), the estimated template (2nd column), and differences between original images and registered template frames (3rd column). 


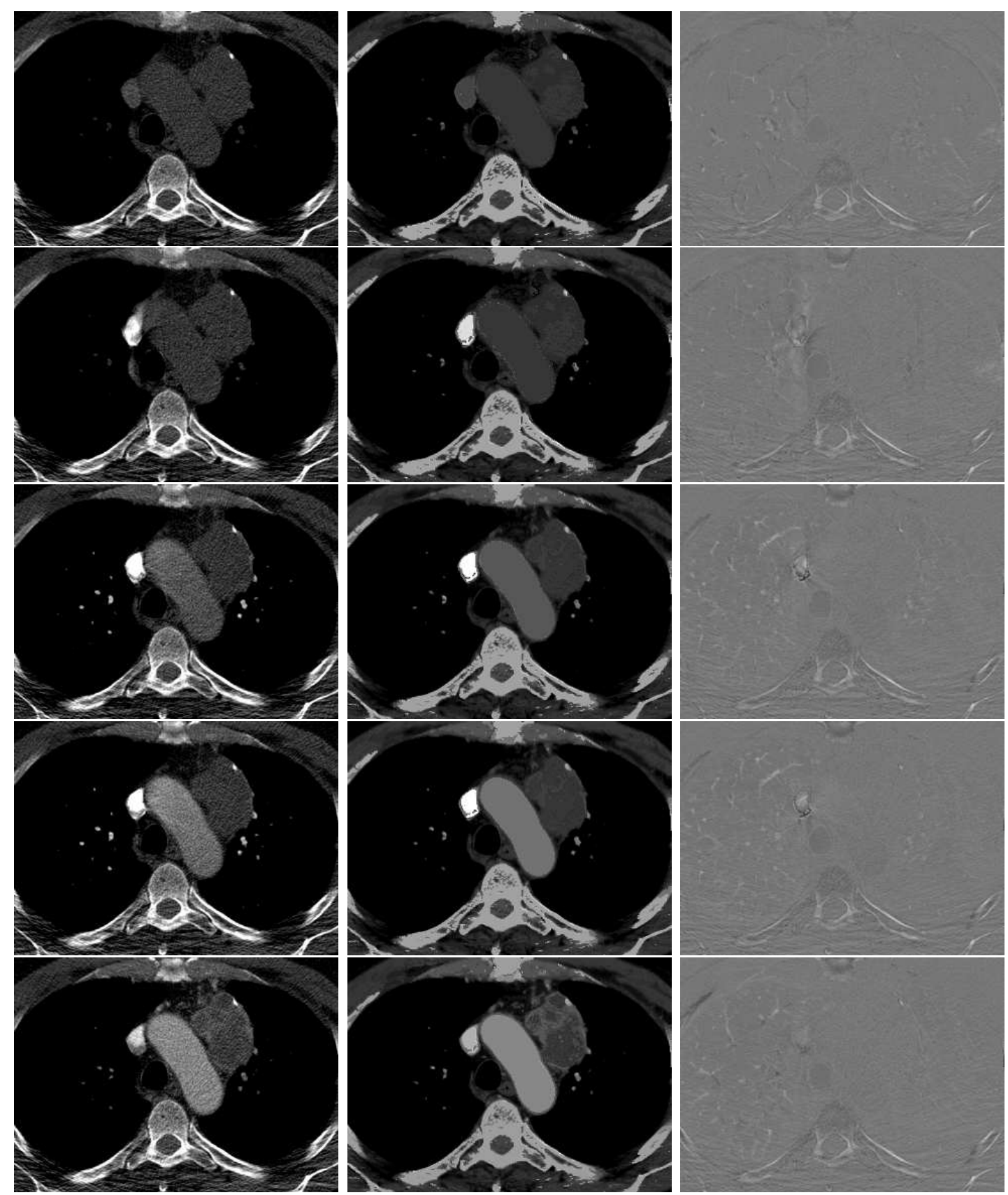

Figure 6. A thoracic DET-CT scan sequence. Some images of the original sequence (1st column), the estimated template (2nd column), and differences between original images and registered template frame (3rd column). 

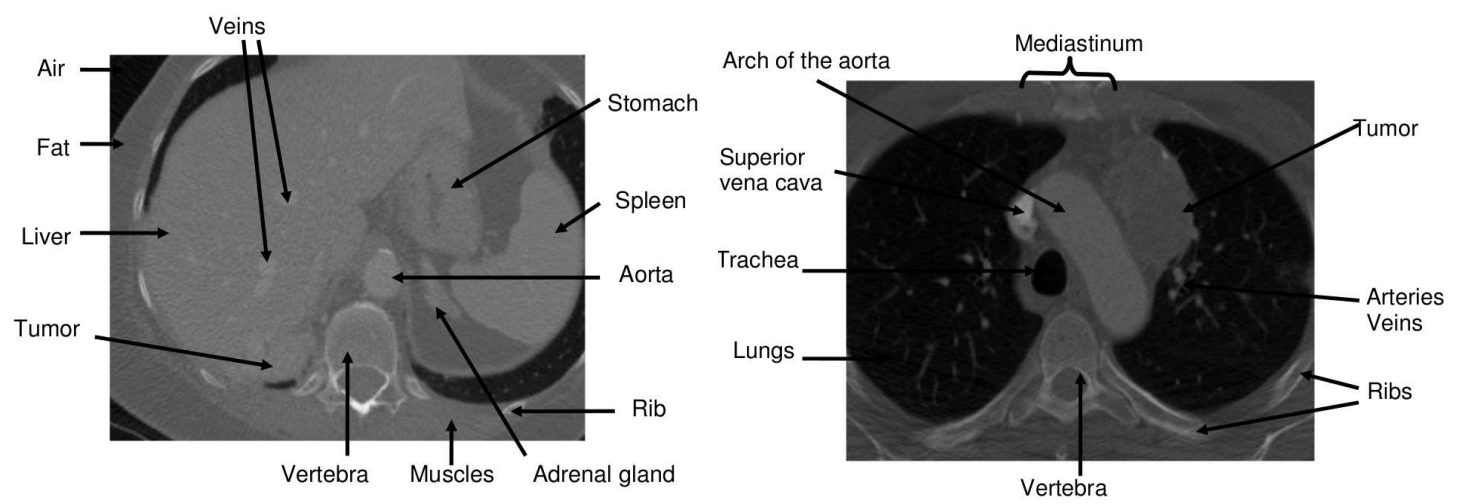

Figure 7. A brief anatomical description of CT-scans of the abdomen and the thorax
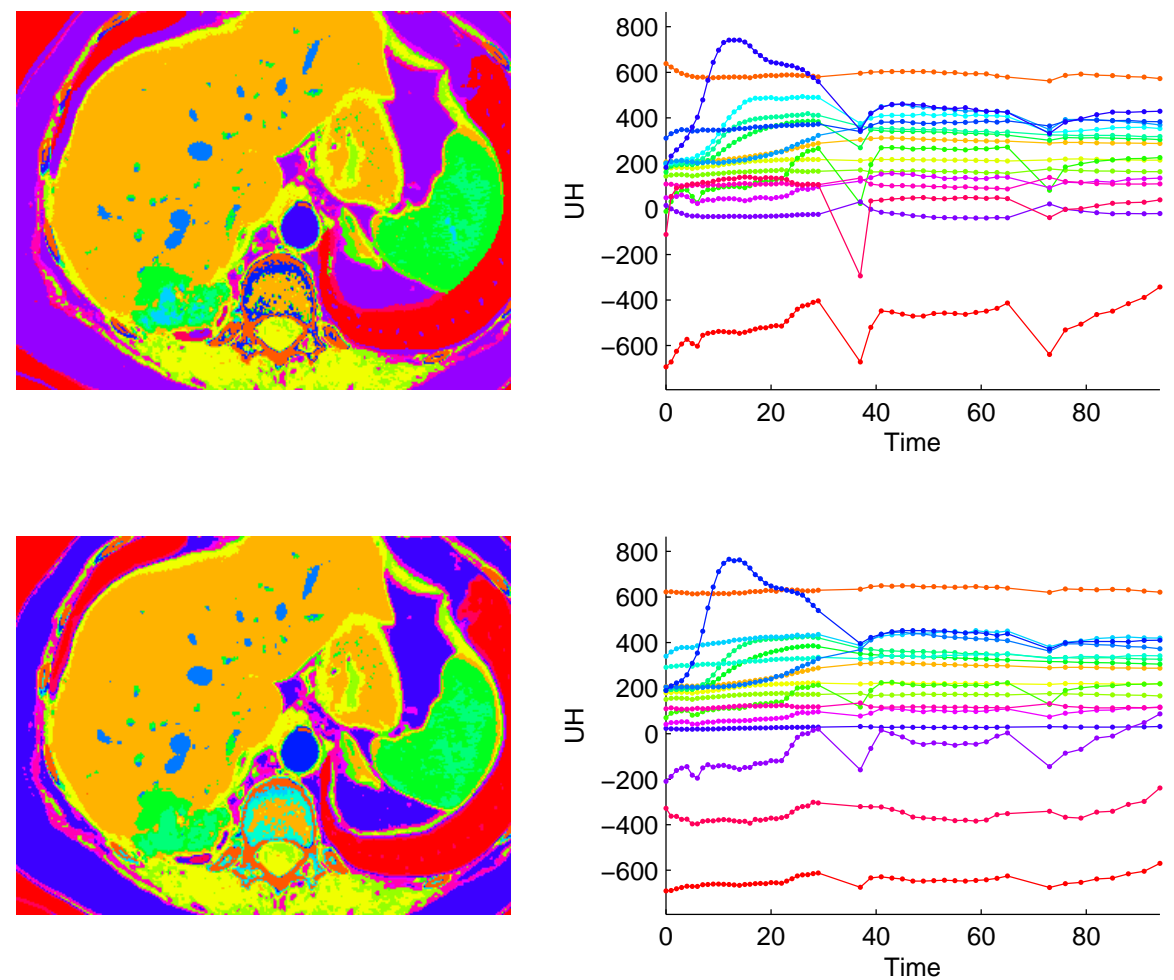

Figure 8. An abdominal sequence: Classifications obtained before (First line) and after registration (Second line), and associated dynamic enhancement curves (UH versus time).

subtile dynamic properties of tissues. Results were approved by radiologists of our team and is currently in use in their hospital. 

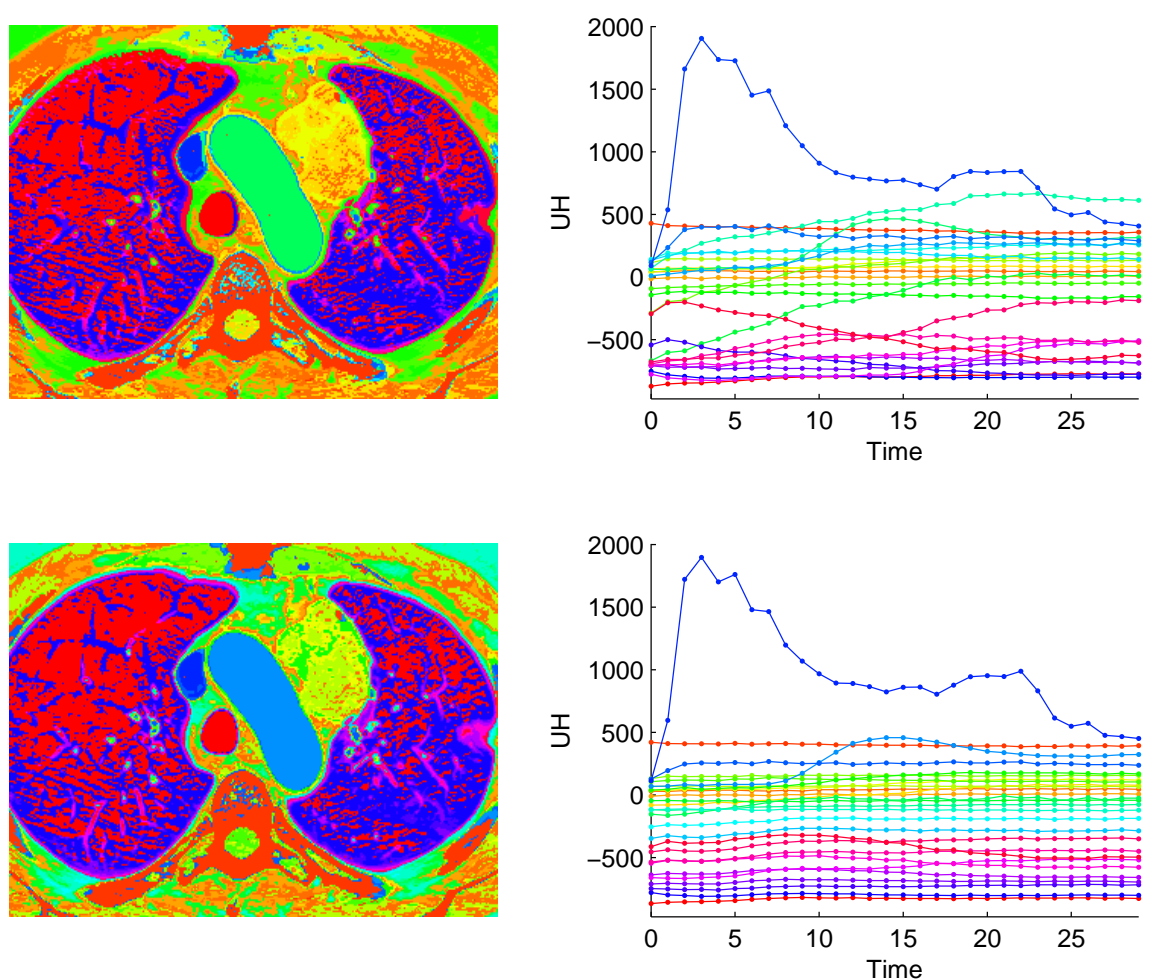

Figure 9. A thoracic sequence: Classifications obtained before (First line) and after registration (Second line), and associated dynamic enhancement curves (UH versus time).

Up to now, we have observed three kinds of improvements due to the registration block of our technique:

- Some inconsistent classes containing unregistered pixels having behaviors similar to real dynamic enhancements were removed after registration (Figure 10),

- some inconsistent classes present before registration were corrected after registration. For instance, after registration of the abdominal sequence, there is a unique class corresponding to fat whereas before registration, the fat class also includes some pixels of the air and the liver located in the upper-left side,

- some new classes appeared after registration. This is particularly the case for the interface classes (see Figures 8 and 9).

On Figures 5 and 6, we show some images of original sequences, together with the estimated temporal templates, and the difference images between original images and registered template frames.

On these images, we notice that temporal templates capture main dynamic enhancement features of the sequences. In the first sequence, the aorta, which takes 

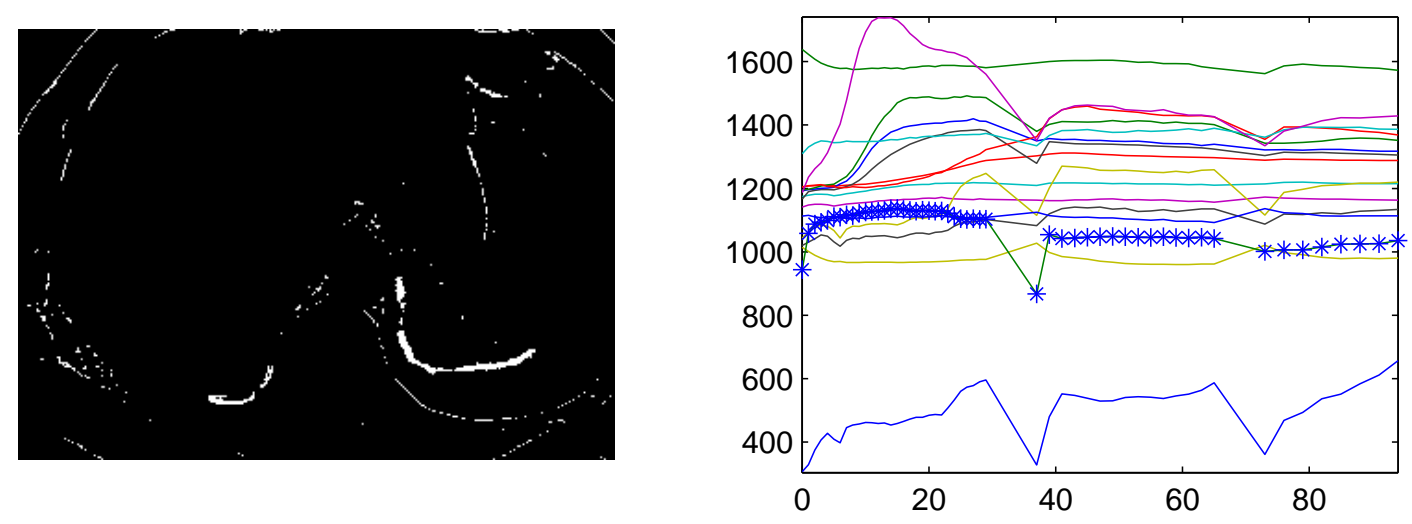

FiguRE 10. Example of a pixel class removed after registration.

contrast first, is followed by the lesion and veins. In the second sequence, vena cava takes contrast first and the arch of aorta is second.

Besides, due to the estimation of contrast curves on classes, the noise present in initial images is filtered and appears attenuated in templates.

The registration reduces significantly differences between initial images and estimated template. However, some differences remain. For instance, on the first sequence, we notice some differences due to topology changes on the right side of vertebra (two separated parts are merged). We also observe some differences in the air located in superior right side of images in both sequences. This is due to the fact that corresponding classes are not homogeneous and have relatively high standard deviation. This is also the case of the superior vena cava on the second sequence.

In order to perform a quantitative evaluation of the registration, we compared the segmentations of several anatomical regions segmented manually on some selected images. For each image sequence, we used the following procedure (see Figure 11). We chose $m$ images from the original sequence $(m=6)$, associated to timest $t_{1}, \ldots, t_{m}$. We form a ground truth by manually segmenting some selected anatomical regions on chosen images. We denote by $\left(S_{i}^{*}\right)_{i=1, m}$ the set of pixels of these segmented regions. By choosing an observation $J^{t_{j}}$, we locate each region $S_{j}^{*}$ in the template by applying registration deformations $\phi^{t_{j}}\left(S_{j}^{*}\right)$. Then, we use inverse registration transformations to compute new segmentations of chosen observed images:

$$
S_{j, i}=\left(\phi^{t_{i}}\right)^{-1}\left(\phi^{t_{j}}\left(S_{j}^{*}\right)\right) \cap \Omega_{d} \quad, i=1, \ldots, m .
$$

The obtained registration-dependent segmentations $\left(S_{j, i}\right)_{i=1}^{m}$ are then compared to the ground truth segmentations $\left(S_{i}^{*}\right)_{i=1}^{m}$ using the number of badly classified pixels $\operatorname{Card}\left(S_{i}^{*} \Delta S_{j, i}\right)$ as a criterion. The overall error is then computed as a mean:

$$
E r r=\frac{1}{m(m-1)} \sum_{i=1}^{m} \sum_{j=1, j \neq i}^{m} \operatorname{Card}\left(S_{i}^{*} \Delta S_{j, i}\right)
$$




\section{- Ground truth ( - ) versus registration-based ( - ) segmentations -}

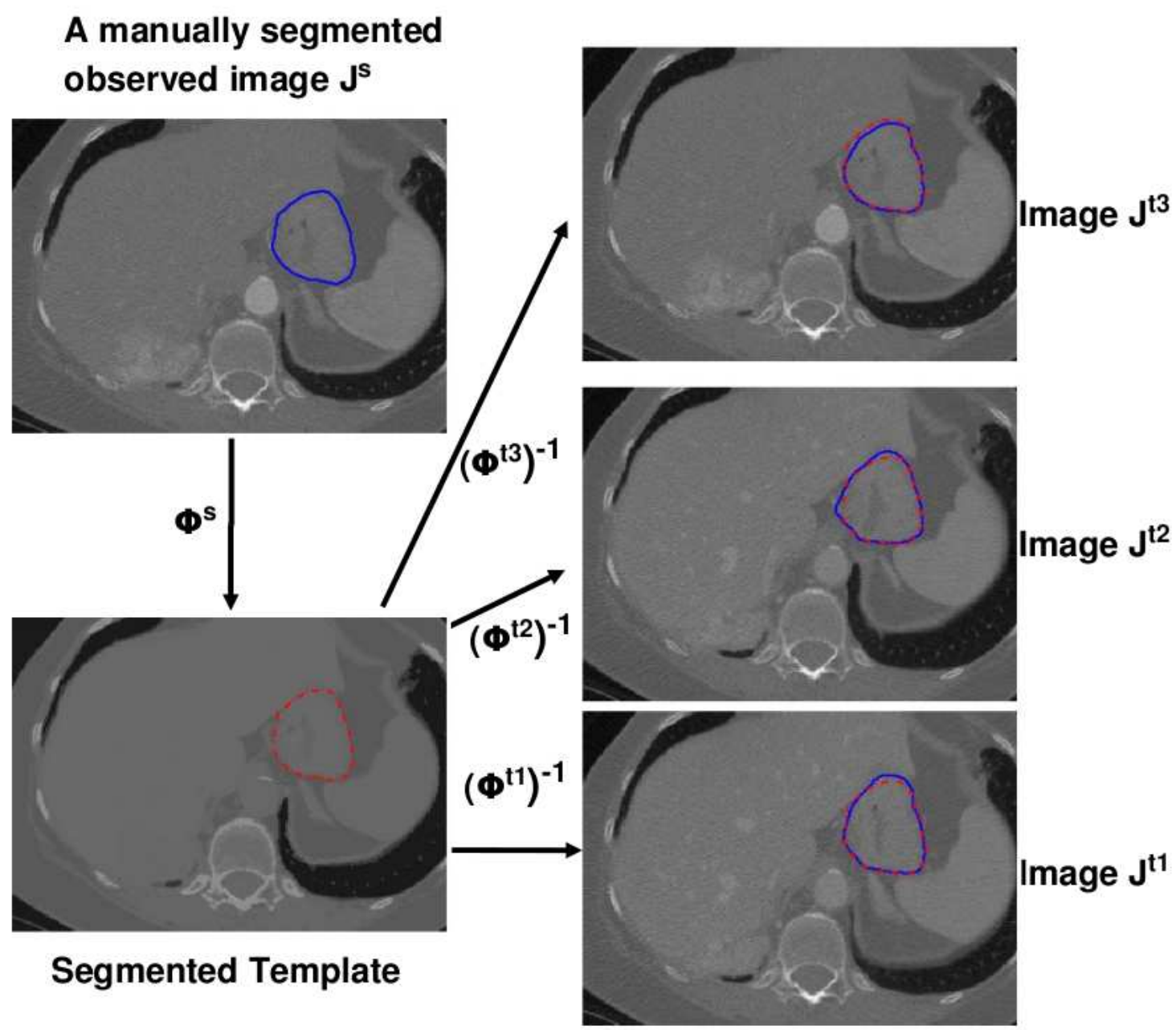

Figure 11. The registration evaluation procedure. A comparison is done between manual and registration-based segmentations. The manual segmentations of a given image is obtained by projecting an initial manual segmentation of another image onto the given image using the registration deformations and the template.

To compare segmentations of regions having different sizes, we also compute the mean of Jaccard coefficient [22]. This criterion, which quantifies the overlap between two sets $X$ et $Y$ by a normalized value $J C$, has a value between 0 et 1 :

$$
J C=\frac{\operatorname{Card} X \cap Y}{\operatorname{Card} X \cup Y} .
$$


So, the overall segmentation quality can also be evaluated by a normalized value $J C_{m}$ which is independent of region size variations

$$
J C_{m}=\frac{1}{m(m-1)} \sum_{i=1}^{m} \sum_{j=1, j \neq i}^{m} \frac{\operatorname{Card}\left(S_{i}^{*} \cap S_{j, i}\right)}{\operatorname{Card}\left(S_{i}^{*} \cup S_{j, i}\right)} .
$$

The registration evaluation is done by comparing segmentation results obtained before and after registration. Results are reported on Tables 1 and 2 .

\begin{tabular}{|c|c|c|c|c|}
\hline & \multicolumn{2}{|c|}{ Err } & \multicolumn{2}{c|}{$J C_{m}$} \\
\hline Region & Before Reg. & After Reg. & Before Reg. & After Reg. \\
\hline Aorta & 50.7 & 54.3 & 0.917 & 0.911 \\
Stomach & 458.4 & 288.1 & 0.865 & 0.912 \\
Liver & 973.6 & 651.6 & 0.964 & 0.976 \\
Lesion & 313.4 & 258.5 & 0.794 & 0.825 \\
Spleen & 739 & 310.8 & 0.865 & 0.940 \\
\hline
\end{tabular}

TABLE 1. A quantitative registration evaluation on the first sequence

\begin{tabular}{|c|c|c|c|c|}
\hline & \multicolumn{2}{|c|}{ Err } & \multicolumn{2}{c|}{$J C_{m}$} \\
\hline Region & Before Reg. & After Reg. & Before Reg. & After Reg. \\
\hline Aorta & 209.3 & 200.7 & 0.950 & 0.952 \\
trachea & 77.5 & 27.5 & 0.859 & 0.946 \\
lungs & 1097.7 & 426.7 & 0.948 & 0.979 \\
tumor & 342.9 & 317.0 & 0.903 & 0.910 \\
\hline
\end{tabular}

TABLE 2. A quantitative registration evaluation on the second sequence.

From Tables 1 and 2, we can say that our new technique improves the segmentation results by reducing the number of badly classified pixels, especially for regions which are significantly moving and characterized by a low Jaccard coefficient before registration. This is specially the case for the spleen, the stomach, and the lesion in the abdominal sequence, and lungs and trachea in the thoracic sequence. On relative stable regions such as the aorta, the new technique give similar segmentation results to those obtained before registration.

\section{Discussion and Conclusion}

The estimation of contrast enhancement curves from DCE sequences is a difficult problem due in particular to possible movements occurring in sequences. The contrast agent and the patient movements generate two different types of image transformations within sequences: whereas the first one causes intensity transformations modifying gray-level values of successive images, the second ones cause deformations changing only the geometry of tissues. Hence, the main difficulty of 
the problem is to distinguish both types of transformations. From a mathematical point of view, this difficulty is related to the fact that the problem of estimating enhancements and registering the sequence at the same time is ill-posed. Without any constraint on possible enhancements nor on deformations, the problem has a wide choice of possible solutions. As a consequence, a geometrical deformation may not be corrected by registration but instead, it can be seen as a contrast change and compensated for by enhancement estimation.

In the construction of our new technique, we use several constraints which aim at avoiding such an error and restricting relevantly the field of possible solutions of the problem. First, enhancement curves are estimated on classes of pixels instead of single pixels. In a class, some unregistered pixels are generally mixed with numerous correctly registered pixels. Hence, their influence on the curve estimation is restricted due to a mean effect on the class. As a consequence, a class-based estimation is less sensitive to mis-registered pixels than usual pixel-based estimations. Due to the estimation on classes, our technique is also much faster than other pixel-based techniques where curves are estimated for each pixel of images [11].

Besides, we carefully introduced some constraints in steps of the classification scheme itself. For splitting classes at each level of the tree classification step, we designed a modified version of the K-means algorithm. In this algorithm, centers of the class are parametrized by some heuristic curves so as to ensure that several features of centers are consistent with those we expect from dynamic enhancements. Moreover, the classification scheme ends with a pruning step which removes some possible inconsistent classes using a simple criterion on the form of class centers. Due to the use of a parametrized K-means and a pruning step, classes obtained at each step of the technique have an anatomical meaning.

As a preliminary step of our work, the technique presented here was implemented in $2 \mathrm{D}$. Hence, the obtained registrations do not correct the vertical movements of the patients. In the future, we plan to fix this drawback. Notice however that a 3D implementation of our technique only requires modifying the registration part to take into account vertical deformations, and is quite straightforward.

\section{REFERENCES}

[1] K.A. Miles. Perfusion CT for the assessment of tumor vascularity: which protocol? The British journal of radiology, 1(76):36-42, 2003.

[2] J.P. O'Connor, A. Jackson, G.J. Parker, and G.C. Jayson. DCE-MRI biomarkers in the clinical evaluation of antiangiogenic and vascular disrupting agents. British journal of Cancer, 2(96):189-195, 2007.

[3] C.A. Cuénod, L. Fournier, D. Balvay, et al. Tumor angiogenesis: pathophysiology and implications for contrast-enhanced MRI and CT assessment. Abdominal imaging, 31(2):188-193, 2006.

[4] C. Kuhl. The current statuts of breast MR imaging. Part I. Choice of technique, image interpretation, diagnostic accuracy, and transfer to clinical practice. Radiology, 244(2):356-378, 2007. 
[5] A.R. Padhani. Dynamic contrast-enhanced MRI in clinical oncology: current status and future directions. Magnetic resonnance imaging, 16(4):407-422, 2002.

[6] M.A. Zahra, K.G. Hollingsworth, E. Sala, et al. Dynamic constrast-enhanced MRI as a predictor of tumour response to radiotherapy. The Lancet Oncology, 8(1):63-74, January 2007.

[7] M. Wintermark. Brain perfusion-CT in acute stroke patients. European Radiology Supplements, 15(4):d28-d31, novembre 2005.

[8] R.M. Judd, C.H. Lugo-Olivieri, et al. Physiological basis of myocardial contrast enhancement in fast magnetic resonance images of 2-day-old reperfused canine infarcts. Circulation, 92(7):1902-1910, 1995.

[9] W.A. Kaiser. Dynamic magnetic resonance breast imaging using a double breast coil: an important step towards routine examination of the breast. Frontiers in European Radiology, 7:39-68, 1990.

[10] J.P. Stack, O. Redmond, M.B. Codd, et al. Breast disease: tissue characterisation with gd-dtpa enhancement profiles. Radiology, 174:491-494, 1990.

[11] P. Hayton, M. Brady, L. Tarassenko, and N. Moore. Analysis of dynamic MR breast images using a model of contrast enhancement. Medical Image Analysis, 1:207-224, 1997.

[12] S. Mussurakis, D.L. Buckley, S.J. Bowsley, et al. Dynamic contrast-enhanced magnetic resonance imaging of the breast combined with pharmacokinetic analysis of gadolinium-DTPA uptake in the diagnosis of local recurrence of early stage breast carcinoma. Investigative Radiology, 30(11):650-662, 1995.

[13] P.S. Tofts. Modeling tracer kinetics in dynamic Gd-DTPA MR imaging. J Magn Reson Imaging, 7(1):91-101, 1997.

[14] G.A. Buonaccorsi, C. Roberts, S. Cheung, Y. Watson, J.P. O'Connor, K. Davies, A. Jackson, G.C. Jayson, and G.J. Parker. Comparison of the performance of tracer kinetic model-driven registration for dynamic contrast enhanced MRI using different models of contrast enhancement. Academic Radiology, 13(9):1112-1123, September 2006.

[15] P.J. Moate, L. Dougherty, M. Schnall, et al. A modified logistic model to describe gadolinium kinetics in breast tumors. Magnetic Resonance Imaging, 22(467-473), 2004.

[16] H.J. Henkjan, M.R. Engelbrecht, and J.O. Barentsz. Accurate estimation of pharmacokinetic contrast-enhanced dynamic MRI parameters of the prostate. Journal of Magnetic Resonance Imaging, 13(4):607-614, 2001.

[17] V.J. Schmid, B.J. Whitcher, G.Z. Yang, et al. Statistical analysis of pharmacokinetic models in dynamic contrast-enhanced magnetic resonance imaging. In Medical Image Computing and Computer Assisted Intervention (MICCAI05), volume 8, pages 886-893, 2005.

[18] A. Roche, A. Malandain, and N. Ayache. Unifying Maximum Likelihood Approaches in Medical Image Registration. International Journal of Computer Vision of Imaging Systems and Technology, 11:71-80, 2000.

[19] N. Rougon, A. Discher, and F. Preteux. Region-driven statistical non-rigid registration: application to model-based segmentation and tracking of the heart in perfusion mri. In Proceedings SPIE Conference on Mathematical Methods in Pattern and Image Analysis, volume 5916, pages 148-159, San Diego, August 2005.

[20] C. Xiaohua, M. Brady, J.L. Lo, and N. Moore. Simultaneous segmentation and registration of contrast-enhanced breast MRI. In Information Processing in Medical Imaging, pages 126-137, 2005.

[21] M. Hachama, A. Desolneux, and F. Richard. Combining registration and abnormality detection in mammography. In Proc. of the Workshop on Biomedical Image Registratilon, pages $178-185,2006$.

[22] P.-N. Tan, M. Steinbach, and V. Kumar. Introduction to Data Mining. Addison-Wesley, 2006. 